\title{
Hadron correlators, spectral functions and thermal dilepton rates from lattice QCD*
}

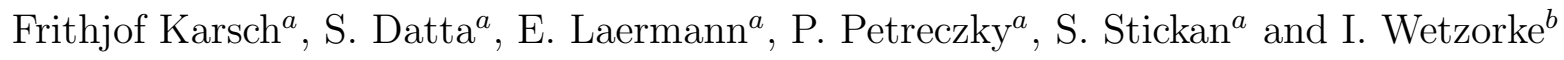 \\ ${ }^{a}$ Fakultät für Physik, Universität Bielefeld, D-33615 Bielefeld, Germany \\ ${ }^{b}$ NIC/DESY Zeuthen, Platanenallee 6, D-15738 Zeuthen, Germany \\ We discuss information on thermal modifications of hadron properties which can be \\ extracted from the structure of Euclidean correlation functions of hadronic currents as well \\ as more direct information obtained through the reconstruction of the spectral functions \\ based on the Maximum Entropy Method.
}

\section{Introduction}

Euclidean time correlation functions, $G_{H}(\tau, T)$, of operators with hadronic quantum numbers $H$ carry all the information about the hadronic spectrum in this quantum number channel, e.g. about the temperature dependence of hadronic masses, the width of these states and eventually also about their disappearance from the spectrum which may happen at high temperature. This information is carried by the spectral function, $\sigma_{H}(\omega, T)$, which is related to $G_{H}(\tau, T)$ through the integral equation,

$G_{H}(\tau, T)=\int_{0}^{\infty} \mathrm{d} \omega \sigma_{H}(\omega, T) \frac{\cosh (\omega(\tau-1 / 2 T))}{\sinh (\omega / 2 T)} \equiv \int_{0}^{\infty} \mathrm{d} \omega \sigma_{H}(\omega, T) K(\omega, T)$.

At $T=0$ the kernel, $K(\omega, T)$, reduces to an exponential function and at large Euclidean times $\tau$ the correlation function picks up the contribution from the lowest lying state contributing to $\sigma_{H}$, i.e. $G_{H}(\tau, T=0) \sim \exp \left(-m_{H} \tau\right)$. At non zero temperature many excited states contribute to the correlation functions, the in-medium spectrum of hadrons gets modified through interactions among hadrons, and an analysis of the asymptotic behaviour of $G_{H}(\tau, T)$ is no longer possible as $\tau$ is limited to the interval $[0,1 / T)$. The reconstruction of $\sigma_{H}(\omega, T)$ and in particular the determination of its low energy structure thus is difficult at non-zero temperature. Additional complications arise in lattice calculations which necessarily are performed on lattices with finite number of points $\left(N_{\tau}\right)$ in Euclidean time. The correlation functions $G_{H}(\tau, T)$ can thus be calculated only at a finite set of Euclidean times, $\tau T=k / N_{\tau}$, with $k=0, . . N_{\tau}-1$. In order to reconstruct the spectral functions from this limited set of information it is necessary to include in the statistical analysis of numerical results also prior information on the structure of $G_{H}(\tau, T)$ as well as assumptions about the likelihood of a certain spectral function $\sigma_{H}(\omega, T)$. It has been suggested to provide this additional information through the application of the Maximum Entropy Method (MEM) [ 1, 2], which has been applied successfully to many

\footnotetext{
*The work has partly been supported by the DFG under grant FOR 339/2-1.
} 

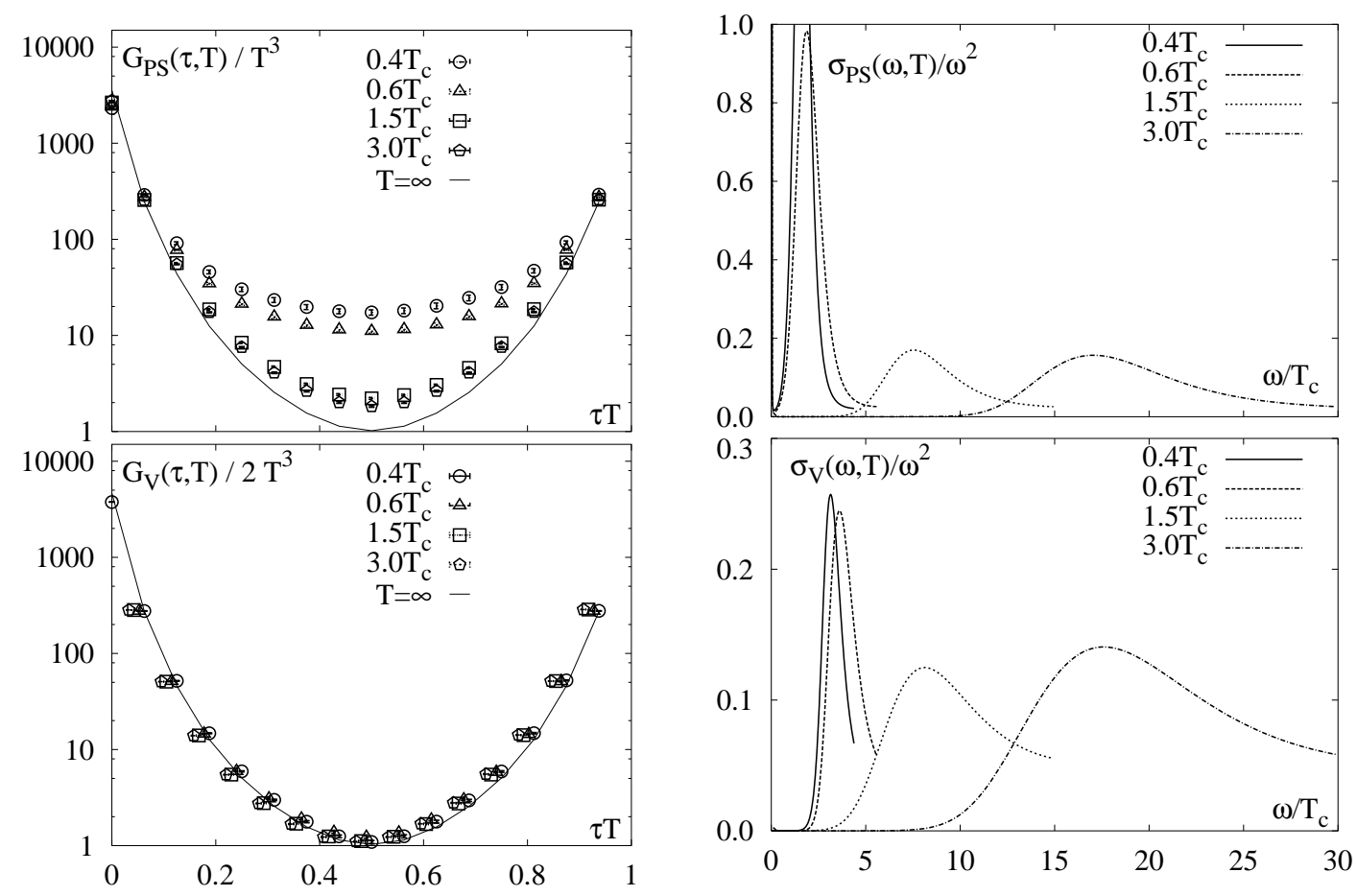

Figure 1. Pseudo-scalar (up) and vector (down) correlation (left) and spectral (right) functions. The solid line in the left hand figures corresponds to the free $(T \rightarrow \infty)$ mesonic correlation function calculated on lattices of same size. For better visibility we have displaced some data for $G_{V}(\tau, T)$ and only show the low energy regime, $\omega / T<10$, for $\sigma_{H}(\omega, T)$. Further details including an error analysis will be presented elsewhere.

other ill-conditioned problems in physics. In the context of QCD it has been shown that the MEM analysis correctly describes spectral properties of Euclidean correlation functions at zero [1, 2] as well as finite [ [3] temperature.

We will discuss here results on thermal properties of light and heavy quark mesonic correlation functions. We stress thermal properties of the correlators, which independent of any statistical analysis provide evidence for changes in the spectral properties, and in addition present results obtained from a MEM analysis of these correlation functions.

\section{Euclidean correlation functions in the scalar and vector channel}

In Fig. 1 1 we show zero-momentum, pseudo-scalar (PS) and vector (V) meson correlation functions calculated at various temperatures on lattices of size $64^{3} \times 16$ in quenched QCD using non-perturbatively improved Wilson fermions. Calculations below $T_{c}$ have been performed at non-vanishing values of the quark mass $\left(m_{q}\right)$ corresponding to a pion mass of about $500 \mathrm{MeV}$ while above $T_{c}$ the calculations have been performed in the chiral limit. The drastic change that occurs in the structure of $G_{H}(\tau, T)$ when crossing the deconfinement transition temperature $T_{c}$ is clearly visible in the PS-channel. We note that this is not related to the different $m_{q}$-values used below and above $T_{c}$. On the contrary, decreasing $m_{q}$ further below $T_{c}$ would lead to even flatter correlation functions indicating 
a decrease in the PS-meson mass. Indeed, a systematic analysis of the $m_{q}$-dependence shows that the lowest excitation in the PS-channel remains massless in the chiral limit for all temperatures below $T_{c}$. Above $T_{c}$ the scalar and pseudo-scalar correlation functions are degenerate, reflecting approximate chiral $\left(U_{A}(1)\right)$ symmetry restoration, and both approach the meson correlation function constructed from freely propagating, massless quarks. However, in particular when we compare $G_{P S}(\tau, T)$ to the vector channel it is evident that it still deviates significantly from this asymptotic form, which is expected to be reached only at infinite temperature. We also note that a free meson correlation function constructed from massive quarks would be steeper than the solid curves shown in the left hand part of Fig. 1. The correlation functions thus indicate that a strong correlation at low energies persists above $T_{c}$ for the propagating $q \bar{q}$-pair.

The drastic change visible in $G_{P S}(\tau, T)$ also gets reflected in the spectral functions reconstructed from this correlation function using MEM. The pronounced $\delta$-function like peak present in the spectral functions below $T_{c}$ gets replaced by a broad "resonance" above $T_{c}$ which moves to larger energies with increasing $T$. In fact, the spectral functions almost coincide above $T_{c}$ when plotted as function of $\omega / T$ (rather than $\omega / T_{c}$ as shown in Fig. (1) which reflects the weak temperature dependence of $G_{P S}(\tau, T)$ above $T_{c}$.

The structure of the spectral functions obtained in the vector channel is similar to those in the pseudo-scalar channel, although the vector correlation function is much closer to the free vector correlation function at all temperatures. In the spectral function this is reflected in the reduced strength of the vector meson pole contribution below $T_{c}$ (note the different scale in the right hand figures) and a less pronounced "resonance" like structure above $T_{c}$.

\section{Thermal dilepton rate}

The vector spectral function shown in Fig. 1 is directly related to the thermal cross section for the production of dilepton pairs at vanishing momentum (see Fig. 2).

$$
\frac{\mathrm{d} W}{\mathrm{~d} \omega \mathrm{d}^{3} p}=\frac{5 \alpha^{2}}{27 \pi^{2}} \frac{1}{\omega^{2}\left(\mathrm{e}^{\omega / T}-1\right)} \sigma_{V}(\omega, \vec{p}, T)
$$

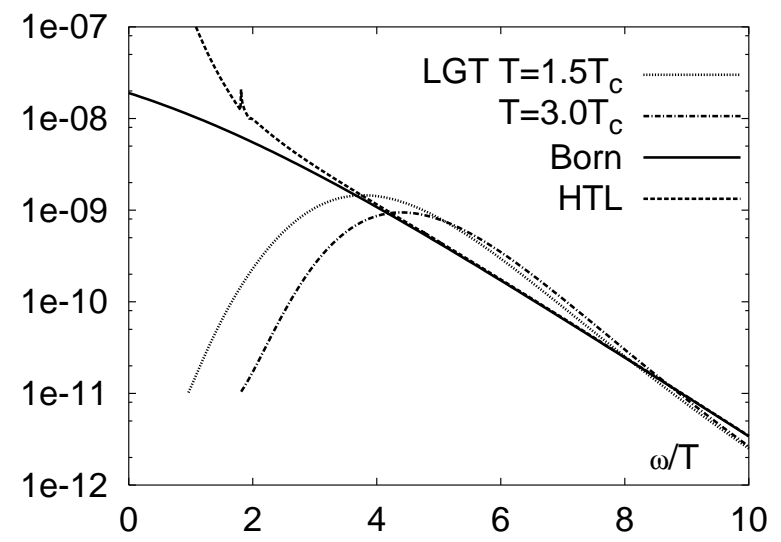

Figure 2. Thermal dilepton cross section determined from the vector spectral function calculated on the lattice as shown in Fig. 1. For comparison we also give the leading order perturbative result (Born) and the result of the HTL-resummed perturbative calculation [4] with a thermal mass, $m_{H T L} / T=g(T) / \sqrt{6}$, chosen as unity. 
This thermal dilepton rate is shown in Fig. 2. The "resonance" like enhancement is visible in this figure as an enhancement of the rate over the perturbative tree level (Born) rate for energies $\omega / T \in[4,8]$. Discrepancies with hard thermal loop calculations [4] show up at smaller energies where lattice results for the spectral functions rapidly drop while the HTL result diverges in the infrared limit. It is to be expected that perturbative as well as lattice calculations do not yet describe this infrared regime correctly and will improve in the future. The fact, that the lattice calculations of $G_{V}(1 / 2 T, T)$ do stay close to the free result, $G_{V}^{\text {free }}(1 / 2 T, T) \equiv 2$, also above $T_{c}$ does, however, put already now a stringent constraint on the properties of $\sigma_{V}(\omega, T)$. In particular, the value of $G_{V}(\tau, T)$ at $\tau T=1 / 2$ is related to a simple, exponentially damped integral over the spectral function,

$\frac{G_{V}(1 / 2 T, T)}{T^{3}} \equiv \int_{0}^{\infty} \mathrm{d} \omega \frac{\sigma_{V}(\omega, T)}{\sinh (\omega / 2 T)}=\left\{\begin{array}{ll}2.23 \pm 0.05 & , T / T_{c}=1.5 \\ 2.21 \pm 0.05 & , T / T_{c}=3\end{array}\right.$.

This relation demands that the spectral function vanishes in the limit $\omega \rightarrow 0$. In fact, in order to get non-vanishing, finite transport coefficients in the QGP the spectral function should be proportional to $\omega$ in this limit [ 5].

\section{Heavy quark bound states at high temperature}

As the properties of mesons constructed from light quarks are closely related to chiral properties of QCD it is expected that these states are strongly influenced by the chiral phase transition to the QGP. The situation, however, is different for heavy quark bound states, which generally are expected to be sensitive to the deconfining aspect of the QCD phase transition. Whether a heavy quark bound state survives the QCD phase transition or not strongly depends on the efficient screening of the interaction among quarks and antiquarks in the QGP. Potential model calculations indicate that some $c \bar{c}$ bound states, e.g. the $J / \psi$, could survive at temperatures close to $T_{c}$ while orbitally excited states like the $\chi_{c}$ most likely get dissolved at $T_{c}$ [ 6]. A more direct analysis of the fate of heavy quark bound states again can come from the analysis of thermal meson correlation functions constructed with heavy quarks. Like in the light quark sector already the temperature dependence of the correlation functions themselves is instructive. The pseudo-scalar $\left(\eta_{c}\right)$ and vector $(J / \psi)$ correlation functions show almost no temperature dependence across the phase transition while the scalar $\left(\chi_{c, 0}\right)$ and axial-vector $\left(\chi_{c, 1}\right)$ change significantly. This also is reflected in the spectral functions extracted from these correlators [ 7]. They suggest that the scalar and axial-vector states dissolve at $T_{c}$ while the pseudo-scalar and vector states still seem to be bound at $1.5 T_{c}$.

\section{REFERENCES}

1. M. Asakawa, T. Hatsuda and Y. Nakahara, Prog. Part. Nucl. Phys. 46 (2001) 459.

2. Y. Nakahara, M. Asakawa and T. Hatsuda, Phys. Rev. D 60 (1999) 091503.

3. F. Karsch et al., Phys. Lett. B 530 (2002) 147.

4. E. Braaten, R.D. Pisarski and T.C. Yuan, Phys. Rev. Lett. 64 (1990) 2242.

5. G. Aarts and J. M. Martinez Resco, JHEP 0204 (2002) 053 and references therein.

6. F. Karsch and H. Satz, Z. Phys. C 51 (1991) 209.

7. S. Datta, F. Karsch, P. Petreczky and I. Wetzorke, hep-lat/0208012. 